\title{
Leaf Traits Variability of Ethiopian Mustard (Brasica Carinata A. Braun) Landraces in their Different Plant Parts
}

\author{
Fekadu Amsalu* \\ Holetta Agricultural Research Center P. O. Box 2003, Addis Ababa, Ethiopia
}

*Corresponding Authors: Fekadu Amsalu, Holetta Agricultural Research Center P. O. Box 2003, Addis Ababa, Ethiopia

\begin{abstract}
The experiment was carried to assess traits of leaf variability in their different plant parts for Ethiopian mustard land races in relation to high quantity and quality of leaf production at Holetta Agricultural research Center, Ethiopia. Therefore forty nine genotypes of Ethiopian mustard land races collected from different agro ecologies were evaluated to assess the traits of leaf variability in their different parts of bottom, middle and top of plant parts for Ethiopian mustard land races in relation to high quantity and quality of leaf production. The experiment was carried out in a simple lattice design. Univariate analysis of variance showed that there were significant differences among genotypes for all leaf parameter in their bottom, middle and top different plant parts of leaf traits except leaf width and leaf area from top plant parts compared. The significant difference indicates the existence of genetic variability reflection in genetic effects of leaf variability traits among the accessions which is important for improvement of these traits. The present study revealed the presence of considerable variability among genotypes for all of leaf traits except leaf width and leaf area from top plant parts assessed in their different plant parts. The significant difference of results indicates that the presence of good opportunity to improve these leaf traits of Ethiopian mustard land races using the tested genotypes.
\end{abstract}

Keywords: Ethiopian mustard, leaf production, variability traits, plant parts

\section{INTRODUCTION}

Ethiopian mustard is believed to be originated in the highlands of the Ethiopian plateau and the adjoining portion of East Africa and the Mediterranean coast (Gomez-Campo and Prakash, 1999).It evolved as a natural cross between B. nigra $(\mathrm{BB})(\mathrm{n}=8)$ and B. oleracea $(\mathrm{CC})(\mathrm{n}=9)$ and underwent further chromosomal doubling $(2 \mathrm{n}=34 ; \mathrm{UN}, 1935)$. It is partially amphidiploids. It is cultivated primarily as leafy vegetable and for oil in the seeds, annual, and grows up to 150 and $200 \mathrm{~cm}$, branched, glabrous to slightly hairy at stem and petiole bases, leaves are alternate and simple. It can adapt to highland areas (2600 msl), with cool climate 0-5 degree centigrade. Cool weather followed by high temperature induces flowering, but decrease leaf production. The crop is traditionally used for many purposes, such as greasing traditional bread-baking clay pan, curing certain diseases and as a source of vegetable relish (Nigussie, 2001). It is the only highland oil seed vegetable crop able to consume by defoliating its leaves or sold to generate income after month of sowing in most near big city parts of the country. In characterization of Ethiopian mustard for vegetative agro-morphological traits Jane Muthoni, (2010) reported as great variation was seen in leaf number per plant, leaf bloom and leaf blade blistering. Crop improvement through plant breeding, thus, occurs through selection operating on genetic variability. Genetic variability is therefore essential for crop improvement. Breeding of mustard crop has emphasized its improvement mostly as an oilseed crop. No major breeding program to improve the leaf vegetable of mustard is known. Identifying leaf variability traits of Ethiopian mustard that is heritable to be reflected as effects on quantity and quality for the mass leaf production is crucial for further investigation of improvement program of the crop. Therefore the present study was, executed with the objective of assessing traits of leaf variability for Ethiopian mustard land races in different plant parts with relation to high quantity and quality of leaf production traits.

\section{Materials ANd Methods}

\subsection{Experimental Site}

The experiment was conducted at Holetta Agricultural Research Center in 2013/2014 cropping season from June to December 2013. Holetta (West Shewa Zone of Oromia Region) is located at latitude $9^{\circ} \mathrm{N}$ 
Leaf Traits Variability of Ethiopian Mustard (Brasica Carinata A. Braun) Landraces in their Different Plant Parts

and longitude $38^{\circ} \mathrm{E}$, altitude of $2400 \mathrm{~m}$ a.s.1 situated 30km West of Addis Ababa. It is one of the representatives of oil seed Brassica growing areas in the central highlands of Ethiopia (Nigussie and Mesfin, 1994). The area has a mean annual rainfall of $1059 \mathrm{~mm}$ and temperatures of $23^{\circ} \mathrm{C}$ (maximum) and $8^{\circ} \mathrm{C}$ (minimum). The soil type is Nitisols with soil ph in the range of 6.0 -7.5(Nigussie and Mesfin, 1994).

\subsection{Description of Test Materials}

A total of forty-nine mustard land races that include one local check and one standard check were used in this study. The majority of the accessions represent the national collection from different major mustard growing regions of the country and that are maintained at Holetta agricultural research Center. The accessions were obtained kindly from Holetta agricultural research center of highland oil crops improvement program. The details of the accessions used in the experiment are given in Table 1.

Table1. List of 49 Ethiopian mustard genotypes used in the study and their origin

\begin{tabular}{|c|c|c|c|}
\hline No. & Accession number & Area of collection & Altitude(m) \\
\hline 1 & PGRC/E 20001 & West Wollega/Arjo & 2420 \\
\hline 2 & " 20002 & Bale Zone/Kitu & 2500 \\
\hline 3 & 20004 & South Gonder/Liba & 1980 \\
\hline 4 & 20005 & SouthGonder/Debretabor & 1830 \\
\hline 5 & 20006 & South Gonder/Debretabor & 1980 \\
\hline 6 & 20007 & North Gonder/Woger/Dabat & 2500 \\
\hline 7 & 20017 & West Gojiam /Awi /Dangila & 1980 \\
\hline 8 & 20056 & West Shewa/Jibatenamecha & 2200 \\
\hline 9 & 20065 & West Shewa/Jibatena mecha & 2200 \\
\hline 10 & 20066 & West Shewa/Ambo & 1950 \\
\hline 11 & 20067 & West Shewa/Ambo & 2010 \\
\hline 12 & 20076 & SNNP/Wenago & 1853 \\
\hline 13 & 20077 & South East Tigray/Inderta & 2000 \\
\hline 14 & 20112 & West Gojam/JabiTehnan & 1980 \\
\hline 15 & 20117 & West Shewa/Jibatnamecha & 2050 \\
\hline 16 & 20127 & West Shewa/chelia & 1700 \\
\hline 17 & 20133 & West Shewa/Menagesha & 2600 \\
\hline 18 & 20134 & West Shewa/Jibat & 2200 \\
\hline 19 & 20146 & West Gojam/Bahirdarzuria & 1980 \\
\hline 20 & 20165 & West Gojiam/Awi/Dangila & 1980 \\
\hline 21 & 20166 & West Gojiam/Awi/Dangila & 1980 \\
\hline 22 & 21008 & Arsi/Gedeb & 2380 \\
\hline 23 & 21012 & West shewa/Dendi & 2900 \\
\hline 24 & 21017 & West Shewa/Gendbert & 2470 \\
\hline 25 & 21026 & West Gojiam Awi/Dangila & 2000 \\
\hline 26 & 21035 & West Gojam/Sekela & 2540 \\
\hline 27 & 21037 & West Gojiam/Awi/Dangila & 2165 \\
\hline 28 & 21068 & Bale/Adaba & 2500 \\
\hline 29 & 21157 & SNNP /South omo & 2830 \\
\hline 30 & 21225 & East Gojam/Enemay & 2000 \\
\hline 31 & 208411 & West Gonder/Debretabor & 2150 \\
\hline 32 & 229665 & West Gojam/Burie & 2050 \\
\hline 33 & 237048 & Arsie-Robe & 2350 \\
\hline 34 & 241907 & South Gonder/Fogera & 1825 \\
\hline 35 & 241910 & South Gonder/Farta & 2289 \\
\hline
\end{tabular}


Leaf Traits Variability of Ethiopian Mustard (Brasica Carinata A. Braun) Landraces in their Different Plant Parts

\begin{tabular}{|c|c|c|c|}
\hline 36 & " 242856 & Arsi zone /Sherka & 2360 \\
\hline 37 & 242858 & Arsi zone /Sherka & 2360 \\
\hline 38 & 243738 & South Wollo/Desiezuria & 2928 \\
\hline 39 & 243739 & South Wollo/Tenta & 2950 \\
\hline 40 & 21256 & West Gojam/Bahirdarzuria & 1940 \\
\hline 41 & 243750 & Wollo/kalu & 2020 \\
\hline 42 & 2243756 & South Gonder/ Debark & 3115 \\
\hline 43 & 243761 & Gonder Zuria & 2050 \\
\hline 44 & 243763 & South Gonder/Kemkem & 2070 \\
\hline 45 & " 208556 & West Shewa/Adis Alem & 2200 \\
\hline 46 & 208585 & East Shewa/yerer & 1600 \\
\hline 47 & Yellow dodolla & Bale/Dodolla & 2500 \\
\hline 48 & (ZemX Yellow Dodolla ) & Cross & 2400 \\
\hline 49 & Local check & Holetta area & 2400 \\
\hline
\end{tabular}

Source: Holetta highland oil crops research program

\subsection{Experimental Design, Management and Season}

The experiment was executed from June 2013 to December 2013. The experiment was laid out in simple lattice design with two replications. A plot of four central rows each three-meter long and $30 \mathrm{~cm}$ spacing between rows were used for data collection. Each replication had seven blocks and each block was represented by seven plots. The path between blocks was $2 \mathrm{~m}$ and the spacing between plots with in sub-blocks was also $0.6 \mathrm{~m}$. Each entry was manually drilled a rate of $10 \mathrm{~kg} / \mathrm{ha}$ and urea and phosphorous fertilizers were applied at the rates of $46 / 69 \mathrm{~kg} / \mathrm{ha} \mathrm{N} / \mathrm{P}_{2} \mathrm{O}_{5}$ respectively following the national recommendations. All other recommended agronomic and cultural practices were carried out following practices described by Adefris (2005).

\subsection{Data Collected}

Data on plant was collected from five plants randomly selected from the central rows of each plot and averaged for statistical analysis.

1. Date of 50\% flowering: total days from sowing to 50\% date of flowering observed.

2. Seeds yield per plot(SYP): Seed yield per plot measured in grams after moisture of the seed was adjusted to 7 percent..

3. Plant height (PHT): The average height of five randomly selected plants was measured in centimeters from the ground surface to the top of the main stem at maturity.

4. Petiole length: Measurements of the petiole length of three leafs of petiole from bottom, middle and top of five plants of leaves excluding leaves.

5. Leaf length $(\mathrm{cm})$ : measurements of the leaf length of three leafs length from bottom, middle and top of five plants. From each leaf starting from the base to the apex of leaf blade excluding petiole was measured at full vegetative stage.

6. Leaf width $(\mathrm{cm})$ : An actual measurement of width of leafs across the widest portion/section of the same leaf from bottom, middle and top of five plants was measured at full vegetative stage.

7. Leaf area: was measured using leaf area meter from bottom, middle and top of five plants for three leaf blade.

\section{RESULTS AND DISCUSSION}

\subsection{Mean Values of Ethiopian mustard Leaf Traits from Bottom, Middle and Top Parts of Plants}

Average mean values of the leaf traits from bottom, middle and top parts of plants and its related traits of the Ethiopian mustard were recorded for date of flowering, seed yield per plot, plant height, petiole length of leaf from bottom, middle and top of plant parts, leaf length from bottom, middle, and top of plant parts, leaf width from bottom, middle and top of plant parts and leaf area from bottom middle and 
top of plant parts are presented in Table 2. Means of date of flowering was greater than 100 days for ten genotypes (PGRC/E20056, PGRC/E20065, PGRC/E20117, PGRC/E20127, PGRC/E 20134, PGRC/E21012, PGRC/E21017, PGRC/E21035, PGRC/E237048 and PGRC/E243756). Mean values of seed yield per plot was highest for the genotype yellow dodola $(3297 \mathrm{~kg} / \mathrm{ha})$ and the least was recorded for the genotypes PGRC/E20065 (904kg/ha).The highest mean values of petiole length of the tested genotypes from bottom, middle and top of plant parts was recorded for the genotypes PGRC/E20134, PGRC/E243756 and PGRCE/E2006 (14.4, 14.4, 7.5, 13. 2, 13. 2, 10. 7, 12.9, 12.9, and $9.2 \mathrm{~cm}$ ) respectively. Similarly the highest average mean values of leaf length of the tested genotypes from bottom, middle and top of plant parts was recorded for the genotypes PGRC/E243756, PGRC/E20134 and PGRCE/E20065(12.4,12.2,9.9,13.2,11.3,8.8,11.1,10.1 and $9.6 \mathrm{~cm})$ respectively. In addition to highest leaf length the wider leaf width of mean value was recorded from bottom, middle and top of plant parts for the genotype PGRC/E20134, PGRC/E229665 and PGRCE/E21035 $(10.5,8.6,5.2,10.3,8.5,5.8,9.6,8.9,6.8 \mathrm{~cm})$ respectively. On the other hand the highest and best of all genotypes tested average mean value of leaf area of genotypes from bottom, middle and top of plant parts was recorded for the genotypes PGRC/E20134, PGRC/E20117 and PGRC/E237048(83.8,72.1,37.5,76.8,58.5,24.0,76.6,51.8,47.0 cm) respectively.

Table2. Mean values of the studied 49 genotypes for 15 leaf traits of Ethiopian mustard tested at Holetta, 2013/14 Holetta, 2013/14

\begin{tabular}{|c|c|c|c|c|c|c|c|c|c|c|c|c|c|c|c|c|}
\hline \multirow[b]{2}{*}{$\begin{array}{l}\mathrm{N} \\
\mathrm{o}\end{array}$} & \multirow[b]{2}{*}{$\begin{array}{l}\text { Treatmen } \\
\text { ts }\end{array}$} & \multirow[b]{2}{*}{$\begin{array}{l}D \\
F\end{array}$} & \multirow[b]{2}{*}{$\begin{array}{l}\text { SY } \\
\text { P }\end{array}$} & \multirow[b]{2}{*}{$\begin{array}{l}\mathrm{P} \\
\mathrm{H}\end{array}$} & \multicolumn{3}{|c|}{$\begin{array}{l}\text { Petiole length from } \\
\text { plant parts of }\end{array}$} & \multicolumn{3}{|c|}{$\begin{array}{l}\text { Leaf length from } \\
\text { plant parts of }\end{array}$} & \multicolumn{4}{|c|}{$\begin{array}{l}\text { Leaf width from plant } \\
\text { parts of }\end{array}$} & \multicolumn{2}{|c|}{$\begin{array}{l}\text { Leaf area from } \\
\text { plant parts of }\end{array}$} \\
\hline & & & & & $\begin{array}{l}\text { bott } \\
\text { om }\end{array}$ & $\begin{array}{l}\text { Mid } \\
\text { dle }\end{array}$ & $\begin{array}{l}\text { To } \\
\mathrm{p}\end{array}$ & $\begin{array}{l}\text { Bott } \\
\text { om }\end{array}$ & $\begin{array}{l}\text { mid } \\
\text { dle }\end{array}$ & $\begin{array}{l}\text { To } \\
\mathrm{p}\end{array}$ & $\begin{array}{l}\text { Bott } \\
\text { om }\end{array}$ & $\begin{array}{l}\text { mid } \\
\text { dle }\end{array}$ & $\begin{array}{l}\text { To } \\
\mathrm{p}\end{array}$ & $\begin{array}{l}\text { botto } \\
\mathrm{m}\end{array}$ & $\begin{array}{l}\text { mid } \\
\text { dle }\end{array}$ & top \\
\hline 1 & $\begin{array}{l}\mathrm{PGRC} / \mathrm{E} \\
20001\end{array}$ & 95 & $\begin{array}{l}20 \\
45\end{array}$ & $\begin{array}{l}15 \\
8\end{array}$ & 11.6 & 11.6 & 4.5 & 12.3 & 10.1 & 9.1 & 8.7 & 6.3 & 2.9 & 42.8 & 28.8 & 9.5 \\
\hline 2 & $\begin{array}{c}" \\
20002\end{array}$ & 81 & $\begin{array}{l}13 \\
34\end{array}$ & $\begin{array}{l}15 \\
3\end{array}$ & 4.4 & 4.4 & 3.6 & 5.3 & 5.5 & 5.7 & 4.0 & 3.3 & 2.6 & 13.4 & 5.9 & 8.1 \\
\hline 3 & $\begin{array}{c}" \\
20004\end{array}$ & 90 & $\begin{array}{l}13 \\
90\end{array}$ & $\begin{array}{l}17 \\
2\end{array}$ & 5.1 & 5.1 & 4.6 & 7.8 & 9.1 & 7.7 & 4.6 & 5.4 & 3.8 & 21.3 & 15.9 & $\begin{array}{l}14 . \\
3\end{array}$ \\
\hline 4 & $\begin{array}{c}" \\
20005\end{array}$ & 86 & $\begin{array}{l}18 \\
05\end{array}$ & $\begin{array}{l}17 \\
8\end{array}$ & 6.8 & 6.8 & 4.6 & 7.4 & 6.7 & 6.3 & 5.6 & 4.8 & 3.5 & 29.9 & 25.0 & $\begin{array}{l}19 . \\
8\end{array}$ \\
\hline 5 & $\begin{array}{c}" \\
20006\end{array}$ & 99 & $\begin{array}{l}17 \\
33\end{array}$ & $\begin{array}{l}19 \\
7\end{array}$ & 11.5 & 11.5 & 4.2 & 11.1 & 9.2 & 6.2 & 9.2 & 7.5 & 2.7 & 59.5 & 43.3 & $\begin{array}{l}16 . \\
5\end{array}$ \\
\hline 6 & $\begin{array}{c}" \\
20007\end{array}$ & 90 & $\begin{array}{l}19 \\
75\end{array}$ & $\begin{array}{l}20 \\
3\end{array}$ & 7.7 & 7.7 & 3.5 & 9.1 & 8.2 & 7.1 & 6.7 & 5.3 & 2.9 & 34.3 & 29.4 & $\begin{array}{l}11 . \\
7\end{array}$ \\
\hline 7 & $\begin{array}{c}" \\
20017\end{array}$ & 94 & $\begin{array}{l}18 \\
66\end{array}$ & $\begin{array}{l}19 \\
0\end{array}$ & 9.0 & 9.0 & 4.9 & 10.4 & 10.1 & 8.4 & 8.0 & 6.6 & 4.1 & 41.4 & 44.8 & $\begin{array}{l}19 . \\
5\end{array}$ \\
\hline 8 & $\begin{array}{r}" 1 \\
20056\end{array}$ & $\begin{array}{l}10 \\
2\end{array}$ & $\begin{array}{l}12 \\
26\end{array}$ & $\begin{array}{l}15 \\
9\end{array}$ & 9.8 & 9.8 & 6.4 & 8.5 & 7.3 & 6.5 & 6.8 & 5.7 & 5.6 & 38.5 & 30.3 & $\begin{array}{l}20 . \\
3\end{array}$ \\
\hline 9 & $\begin{array}{c}" \\
20065\end{array}$ & $\begin{array}{l}11 \\
3\end{array}$ & $\begin{array}{l}90 \\
4\end{array}$ & $\begin{array}{l}11 \\
5\end{array}$ & 12.9 & 12.9 & 9.2 & 11.1 & 10.1 & 9.6 & 7.9 & 7.4 & 5.1 & 48.7 & 55.7 & $\begin{array}{l}29 . \\
1\end{array}$ \\
\hline $\begin{array}{l}1 \\
0\end{array}$ & $\begin{array}{c}" \\
20066\end{array}$ & 91 & $\begin{array}{l}13 \\
75\end{array}$ & $\begin{array}{l}18 \\
4\end{array}$ & 12.2 & 12.2 & 8.5 & 11.5 & 10.6 & 9.3 & 9.2 & 8.4 & 6.5 & 68.3 & 64.0 & $\begin{array}{l}31 . \\
0\end{array}$ \\
\hline $\begin{array}{l}1 \\
1\end{array}$ & $\begin{array}{c}" \\
20067\end{array}$ & 89 & $\begin{array}{l}25 \\
34\end{array}$ & $\begin{array}{l}19 \\
8\end{array}$ & 6.7 & 6.7 & 4.6 & 7.4 & 8.1 & 7.5 & 5.1 & 5.7 & 3.4 & 20.7 & 20.5 & $\begin{array}{l}14 . \\
4\end{array}$ \\
\hline $\begin{array}{l}1 \\
2\end{array}$ & $\begin{array}{c}" \\
20076\end{array}$ & 94 & $\begin{array}{l}25 \\
80\end{array}$ & $\begin{array}{l}17 \\
3\end{array}$ & 10.5 & 10.5 & 4.5 & 11.0 & 9.4 & 7.8 & 7.9 & 6.1 & 2.9 & 47.4 & 26.6 & 8.8 \\
\hline $\begin{array}{l}1 \\
3\end{array}$ & $\begin{array}{c}" \\
20077\end{array}$ & 85 & $\begin{array}{l}18 \\
83\end{array}$ & $\begin{array}{l}17 \\
4\end{array}$ & 7.7 & 7.7 & 4.2 & 9.1 & 7.1 & 7.0 & 6.1 & 3.7 & 2.4 & 22.3 & 10.9 & 8.1 \\
\hline $\begin{array}{l}1 \\
4\end{array}$ & $\begin{array}{c}" \\
20112\end{array}$ & 98 & $\begin{array}{l}24 \\
06\end{array}$ & $\begin{array}{l}19 \\
9\end{array}$ & 8.3 & 8.3 & 4.7 & 8.9 & 9.7 & 7.9 & 8.1 & 7.6 & 5.2 & 50.2 & 53.2 & $\begin{array}{l}26 . \\
7\end{array}$ \\
\hline $\begin{array}{l}1 \\
5\end{array}$ & $\begin{array}{c}" \\
20117\end{array}$ & $\begin{array}{l}10 \\
4\end{array}$ & $\begin{array}{l}20 \\
80\end{array}$ & $\begin{array}{l}17 \\
0\end{array}$ & 11.8 & 11.8 & 5.7 & 11.9 & 9.7 & 7.3 & 8.8 & 7.7 & 4.5 & 76.8 & 58.5 & $\begin{array}{l}24 . \\
0\end{array}$ \\
\hline $\begin{array}{l}1 \\
6\end{array}$ & $\begin{array}{c}" \\
20127\end{array}$ & $\begin{array}{l}10 \\
7\end{array}$ & $\begin{array}{l}18 \\
46 \\
\end{array}$ & $\begin{array}{l}17 \\
3\end{array}$ & 12.3 & 12.3 & 7.7 & 11.9 & 10.6 & 9.2 & 9.0 & 7.8 & 5.4 & 58.1 & 52.7 & $\begin{array}{l}38 . \\
0\end{array}$ \\
\hline $\begin{array}{l}1 \\
7\end{array}$ & $\begin{array}{c}" \\
20133\end{array}$ & 85 & $\begin{array}{l}99 \\
9\end{array}$ & $\begin{array}{l}15 \\
4\end{array}$ & 4.4 & 4.4 & 2.6 & 6.2 & 6.4 & 4.9 & 4.1 & 3.5 & 1.9 & 14.3 & 9.3 & 7.2 \\
\hline
\end{tabular}


Leaf Traits Variability of Ethiopian Mustard (Brasica Carinata A. Braun) Landraces in their Different Plant Parts

\begin{tabular}{|c|c|c|c|c|c|c|c|c|c|c|c|c|c|c|c|c|}
\hline $\begin{array}{l}1 \\
8 \\
\end{array}$ & $\begin{array}{c}" \\
20134 \\
\end{array}$ & $\begin{array}{l}10 \\
2 \\
\end{array}$ & $\begin{array}{l}24 \\
73 \\
\end{array}$ & $\begin{array}{l}20 \\
9 \\
\end{array}$ & 14.4 & 14.4 & 7.5 & 13.2 & 11.3 & 8.8 & 10.5 & 8.6 & 5.2 & 83.8 & 72.1 & $\begin{array}{l}37 . \\
5 \\
\end{array}$ \\
\hline $\begin{array}{l}1 \\
9\end{array}$ & $\begin{array}{c}" \\
20146\end{array}$ & 92 & $\begin{array}{l}23 \\
60\end{array}$ & $\begin{array}{l}18 \\
1\end{array}$ & 7.8 & 7.8 & 3.5 & 9.9 & 9.2 & 7.4 & 7.6 & 6.1 & 3.9 & 36.6 & 32.3 & $\begin{array}{l}16 . \\
9\end{array}$ \\
\hline $\begin{array}{l}2 \\
0\end{array}$ & $\begin{array}{r}" \\
20165\end{array}$ & 97 & $\begin{array}{l}24 \\
65\end{array}$ & $\begin{array}{l}21 \\
9\end{array}$ & 10.4 & 10.4 & 5.6 & 11.8 & 10.5 & 8.6 & 8.9 & 7.7 & 4.8 & 68.5 & 43.6 & $\begin{array}{l}29 . \\
7\end{array}$ \\
\hline $\begin{array}{l}2 \\
1\end{array}$ & $\begin{array}{c}" \\
20166\end{array}$ & 94 & $\begin{array}{l}23 \\
62\end{array}$ & $\begin{array}{l}21 \\
0\end{array}$ & 8.8 & 8.8 & 4.0 & 10.1 & 9.5 & 6.4 & 7.3 & 6.7 & 3.3 & 36.4 & 36.8 & $\begin{array}{l}13 . \\
3\end{array}$ \\
\hline $\begin{array}{l}2 \\
2 \\
\end{array}$ & $\begin{array}{c}" \\
21008\end{array}$ & 82 & $\begin{array}{l}16 \\
37 \\
\end{array}$ & $\begin{array}{l}15 \\
9\end{array}$ & 5.7 & 5.7 & 2.6 & 6.8 & 6.2 & 4.7 & 4.5 & 3.1 & 2.2 & 15.4 & 14.4 & 5.4 \\
\hline $\begin{array}{l}2 \\
3\end{array}$ & $\begin{array}{c}" \\
21012\end{array}$ & $\begin{array}{l}10 \\
2\end{array}$ & $\begin{array}{l}21 \\
62\end{array}$ & $\begin{array}{l}14 \\
7\end{array}$ & 9.7 & 9.7 & 5.9 & 9.5 & 8.5 & 7.3 & 7.6 & 6.7 & 4.8 & 43.2 & 39.9 & $\begin{array}{l}21 . \\
7\end{array}$ \\
\hline $\begin{array}{l}2 \\
4\end{array}$ & $\begin{array}{r}" \\
21017\end{array}$ & $\begin{array}{l}10 \\
5\end{array}$ & $\begin{array}{l}22 \\
40\end{array}$ & $\begin{array}{l}17 \\
9\end{array}$ & 11.8 & 11.8 & 6.9 & 10.6 & 10.0 & 7.6 & 8.7 & 7.3 & 4.7 & 47.6 & 57.7 & $\begin{array}{l}19 . \\
1\end{array}$ \\
\hline $\begin{array}{l}2 \\
5\end{array}$ & $\begin{array}{c}" \\
21026\end{array}$ & 99 & $\begin{array}{l}30 \\
54\end{array}$ & $\begin{array}{l}19 \\
3\end{array}$ & 8.4 & 8.4 & 5.2 & 9.5 & 9.5 & 8.1 & 7.8 & 7.2 & 5.1 & 51.2 & 55.8 & $\begin{array}{l}29 . \\
9\end{array}$ \\
\hline $\begin{array}{l}2 \\
6\end{array}$ & $\begin{array}{r}" \\
21035\end{array}$ & $\begin{array}{l}10 \\
6\end{array}$ & $\begin{array}{l}16 \\
43 \\
\end{array}$ & $\begin{array}{l}19 \\
4\end{array}$ & 12.8 & 12.8 & $\begin{array}{l}10 . \\
3\end{array}$ & 12.3 & 11.6 & 8.8 & 9.6 & 8.9 & 6.8 & 50.9 & 57.7 & $\begin{array}{l}47 . \\
1\end{array}$ \\
\hline $\begin{array}{l}2 \\
7 \\
\end{array}$ & $\begin{array}{r}" \\
21037 \\
\end{array}$ & 94 & $\begin{array}{l}26 \\
39 \\
\end{array}$ & $\begin{array}{l}22 \\
4\end{array}$ & 11.1 & 11.1 & 7.9 & 11.4 & 10.2 & 8.2 & 8.9 & 7.2 & 5.3 & 66.3 & 48.5 & $\begin{array}{l}28 . \\
3\end{array}$ \\
\hline $\begin{array}{l}2 \\
8\end{array}$ & $\begin{array}{c}" \\
21068\end{array}$ & 79 & $\begin{array}{l}18 \\
04\end{array}$ & $\begin{array}{l}16 \\
7\end{array}$ & 3.2 & 3.2 & 2.3 & 4.8 & 4.6 & 4.4 & 2.3 & 2.5 & 1.6 & 5.8 & 6.0 & 3.1 \\
\hline $\begin{array}{l}2 \\
9 \\
\end{array}$ & $\begin{array}{r}" \\
21157\end{array}$ & 90 & $\begin{array}{l}13 \\
42 \\
\end{array}$ & $\begin{array}{l}14 \\
4\end{array}$ & 12.6 & 12.6 & 7.2 & 12.4 & 10.9 & 8.0 & 9.3 & 8.1 & 5.5 & 69.8 & 65.8 & $\begin{array}{l}27 . \\
5\end{array}$ \\
\hline $\begin{array}{l}3 \\
0\end{array}$ & $\begin{array}{c}" \\
21225\end{array}$ & 79 & $\begin{array}{l}19 \\
28\end{array}$ & $\begin{array}{l}16 \\
0\end{array}$ & 3.4 & 3.4 & 2.6 & 5.4 & 6.3 & 6.2 & 2.9 & 2.6 & 2.2 & 9.0 & 10.4 & 8.1 \\
\hline $\begin{array}{l}3 \\
1\end{array}$ & $\begin{array}{c}" \\
208411\end{array}$ & $\begin{array}{l}10 \\
0\end{array}$ & $\begin{array}{l}15 \\
86\end{array}$ & $\begin{array}{l}17 \\
9\end{array}$ & 8.7 & 8.7 & 4.7 & 9.3 & 7.9 & 7.0 & 6.9 & 5.3 & 3.7 & 37.4 & 29.9 & $\begin{array}{l}18 . \\
3\end{array}$ \\
\hline $\begin{array}{l}3 \\
2\end{array}$ & $\begin{array}{c}" \\
229665\end{array}$ & $\begin{array}{l}10 \\
0\end{array}$ & $\begin{array}{l}19 \\
04\end{array}$ & $\begin{array}{l}19 \\
4\end{array}$ & 11.1 & 11.1 & 5.7 & 13.0 & 10.9 & 9.3 & 10.3 & 8.5 & 5.8 & 59.0 & 63.7 & $\begin{array}{l}34 . \\
2\end{array}$ \\
\hline $\begin{array}{l}3 \\
3\end{array}$ & $\begin{array}{c}" \\
237048\end{array}$ & $\begin{array}{l}10 \\
9\end{array}$ & $\begin{array}{l}16 \\
88\end{array}$ & $\begin{array}{l}20 \\
1\end{array}$ & 11.6 & 11.6 & 9.9 & 11.6 & 10.5 & $\begin{array}{l}10 . \\
4\end{array}$ & 8.9 & 8.1 & 7.4 & 76.6 & 51.8 & $\begin{array}{l}47 . \\
0\end{array}$ \\
\hline $\begin{array}{l}3 \\
4\end{array}$ & $\begin{array}{c}" \\
241907\end{array}$ & 93 & $\begin{array}{l}19 \\
88\end{array}$ & $\begin{array}{l}19 \\
5\end{array}$ & 7.5 & 7.5 & 4.8 & 8.2 & 8.9 & 7.3 & 6.6 & 6.2 & 4.3 & 44.4 & 26.7 & $\begin{array}{l}19 . \\
4\end{array}$ \\
\hline $\begin{array}{l}3 \\
5\end{array}$ & $\begin{array}{c}" \\
241910\end{array}$ & 93 & $\begin{array}{l}25 \\
66\end{array}$ & $\begin{array}{l}18 \\
7\end{array}$ & 8.7 & 8.7 & 4.7 & 8.5 & 8.4 & 7.1 & 6.9 & 6.2 & 3.4 & 29.0 & 31.5 & $\begin{array}{l}11 . \\
4\end{array}$ \\
\hline $\begin{array}{l}3 \\
6\end{array}$ & $\begin{array}{c}" \\
242856\end{array}$ & 90 & $\begin{array}{l}21 \\
35\end{array}$ & $\begin{array}{l}17 \\
4\end{array}$ & 8.0 & 8.0 & 3.9 & 8.8 & 7.8 & 6.5 & 6.1 & 4.6 & 2.7 & 32.1 & 21.5 & $\begin{array}{l}10 . \\
8\end{array}$ \\
\hline $\begin{array}{l}3 \\
7\end{array}$ & $\begin{array}{c}" \\
242858\end{array}$ & 97 & $\begin{array}{l}20 \\
19\end{array}$ & $\begin{array}{l}20 \\
4\end{array}$ & 12.4 & 12.4 & 7.6 & 11.0 & 9.4 & 8.0 & 7.8 & 6.8 & 4.1 & 48.7 & 42.6 & $\begin{array}{l}25 . \\
1\end{array}$ \\
\hline $\begin{array}{l}3 \\
8\end{array}$ & $\begin{array}{c}" \\
243738\end{array}$ & 88 & $\begin{array}{l}20 \\
22\end{array}$ & $\begin{array}{l}18 \\
8\end{array}$ & 5.8 & 5.8 & 2.9 & 7.7 & 6.9 & 5.7 & 5.0 & 3.9 & 2.1 & 22.9 & 15.5 & 9.3 \\
\hline $\begin{array}{l}3 \\
9\end{array}$ & $\begin{array}{c}" \\
243739\end{array}$ & 86 & $\begin{array}{l}22 \\
31\end{array}$ & $\begin{array}{l}17 \\
8\end{array}$ & 5.4 & 5.4 & 3.9 & 6.3 & 5.1 & 5.6 & 4.2 & 3.6 & 2.4 & 44.5 & 44.6 & $\begin{array}{l}23 . \\
9\end{array}$ \\
\hline $\begin{array}{l}4 \\
0\end{array}$ & $\begin{array}{c}" \\
21256\end{array}$ & 98 & $\begin{array}{l}22 \\
91\end{array}$ & $\begin{array}{l}22 \\
1\end{array}$ & 11.0 & 11.0 & 8.8 & 12.4 & 11.9 & 9.0 & 8.4 & 9.1 & 6.9 & 23.4 & 29.9 & $\begin{array}{l}30 . \\
5\end{array}$ \\
\hline $\begin{array}{l}4 \\
1\end{array}$ & $\begin{array}{c}" \\
243750\end{array}$ & 85 & $\begin{array}{l}21 \\
42\end{array}$ & $\begin{array}{l}19 \\
3\end{array}$ & 7.1 & 7.1 & 3.4 & 8.4 & 7.2 & 6.5 & 5.8 & 4.1 & 3.2 & 27.0 & 22.0 & $\begin{array}{l}13 . \\
6\end{array}$ \\
\hline $\begin{array}{l}4 \\
2\end{array}$ & $\begin{array}{c}" \\
243756\end{array}$ & $\begin{array}{l}10 \\
8\end{array}$ & $\begin{array}{l}18 \\
59\end{array}$ & $\begin{array}{l}18 \\
6\end{array}$ & 13.2 & 13.2 & $\begin{array}{l}10 . \\
3\end{array}$ & 12.4 & 11.2 & 9.9 & 9.9 & 8.9 & 7.7 & 63.3 & 63.1 & $\begin{array}{l}42 . \\
0\end{array}$ \\
\hline $\begin{array}{l}4 \\
3\end{array}$ & $\begin{array}{c}" \\
243761\end{array}$ & 88 & $\begin{array}{l}19 \\
31\end{array}$ & $\begin{array}{l}16 \\
3\end{array}$ & 12.5 & 12.5 & 5.6 & 11.5 & 8.2 & 7.0 & 8.9 & 6.6 & 4.4 & 52.0 & 37.8 & $\begin{array}{l}16 . \\
2\end{array}$ \\
\hline $\begin{array}{l}4 \\
4\end{array}$ & $\begin{array}{c}" \\
243763\end{array}$ & 95 & $\begin{array}{l}13 \\
48\end{array}$ & $\begin{array}{l}16 \\
5\end{array}$ & 9.6 & 9.6 & 4.0 & 9.1 & 8.1 & 5.5 & 7.2 & 6.3 & 2.8 & 36.6 & 28.2 & $\begin{array}{l}12 . \\
6\end{array}$ \\
\hline $\begin{array}{l}4 \\
5\end{array}$ & $\begin{array}{c}" \\
208556\end{array}$ & 86 & $\begin{array}{l}18 \\
79\end{array}$ & $\begin{array}{l}17 \\
2\end{array}$ & 6.7 & 6.7 & 5.6 & 7.7 & 7.6 & 7.0 & 5.8 & 5.0 & 4.4 & 26.4 & 17.6 & $\begin{array}{l}19 . \\
3\end{array}$ \\
\hline $\begin{array}{l}4 \\
6\end{array}$ & $\begin{array}{c}" \\
208585\end{array}$ & 94 & $\begin{array}{l}21 \\
42\end{array}$ & $\begin{array}{l}20 \\
8\end{array}$ & 11.9 & 11.9 & 7.1 & 10.9 & 11.0 & 8.0 & 9.7 & 8.0 & 5.7 & 48.1 & 42.6 & $\begin{array}{l}16 . \\
4\end{array}$ \\
\hline $\begin{array}{l}4 \\
7\end{array}$ & $\begin{array}{l}\text { Yellow } \\
\text { dodolla }\end{array}$ & 85 & $\begin{array}{l}32 \\
97 \\
\end{array}$ & $\begin{array}{l}17 \\
7\end{array}$ & 6.6 & 6.6 & 3.8 & 7.2 & 7.2 & 6.3 & 5.5 & 4.4 & 3.2 & 16.7 & 11.2 & $\begin{array}{l}11 . \\
8\end{array}$ \\
\hline
\end{tabular}


Leaf Traits Variability of Ethiopian Mustard (Brasica Carinata A. Braun) Landraces in their Different Plant Parts

\begin{tabular}{|l|l|l|l|l|l|l|l|l|l|l|l|l|l|l|l|l|}
\hline $\begin{array}{l}4 \\
8\end{array}$ & $\begin{array}{l}\text { ZemXY } \\
\text { D ) }\end{array}$ & 80 & $\begin{array}{l}26 \\
03\end{array}$ & $\begin{array}{l}20 \\
3\end{array}$ & 6.4 & 6.4 & 3.4 & 8.8 & 6.9 & 6.1 & 5.9 & 3.7 & 3.0 & 28.2 & 13.7 & $\begin{array}{l}10 . \\
2\end{array}$ \\
\hline $\begin{array}{l}4 \\
9\end{array}$ & $\begin{array}{l}\text { Local } \\
\text { check }\end{array}$ & 85 & $\begin{array}{l}22 \\
12\end{array}$ & $\begin{array}{l}15 \\
5\end{array}$ & 4.9 & 4.9 & 3.2 & 6.0 & 5.9 & 6.1 & 4.1 & 3.4 & 2.7 & 10.6 & 9.0 & 7.5 \\
& Mean & 93 & $\begin{array}{l}19 \\
99\end{array}$ & $\begin{array}{l}18 \\
1\end{array}$ & 8.9 & 7.4 & 5.3 & 9.5 & 8.7 & 7.3 & 7.11 & 6.01 & 4.0 & 40.77 & 35.0 & $\begin{array}{l}19 . \\
7\end{array}$ \\
& & & & 3 & & & & & & & \\
7 & \\
& CV (\%) & 3. & 24. & 7. & 20.3 & 22.6 & 37. & 18.2 & 18.3 & 24. & 20.7 & 22.8 & 42. & 39.96 & 46.4 & 71. \\
08 & 38 & 0 & 7 & 99 & 2 & 9 & 31 & 8 & 6 & 62 & & 9 & 96 \\
\hline & LSD & 6. & & 29 & 3.7 & 3.4 & 4.1 & 3.5 & 3.2 & 3.6 & 2.9 & 2.7 & 3.5 & 32.7 & 34.9 & 28. \\
& $(0.05)$ & 34 & & .1 & & & & & & & & & & & & 7 \\
\hline
\end{tabular}

The analysis of variance for the 15 leaf traits studied is given in Table 3. The analysis of variance showed that there were significant differences among genotypes for all leaf parameter in their bottom, middle and top different plant parts of leaf traits except leaf width and leaf area from top plant parts compared. Among analyzed leaf traits highly and significantly differences were observed in date of $50 \%$ flowering, plant height, petiole length from bottom plant parts, petiole length from middle plant parts, petiole length from top plant parts, leaf length from bottom parts of plants, leaf length from middle parts of plants, leaf length from top parts of plants , leaf width from bottom parts of plants, leaf width from middle parts of plants, leaf area from bottom parts of plants, leaf area from middle parts of plants traits. The significant difference indicates the existence of genetic variability among the accessions that is important for selection and breeding. Rabbani et al. studied 52 mustard accessions for various both agronomic and physiological interests. Revilla and Tracy found considerable level of diversity among their executed experiment.

Table3. Mean squares for different sources of variations for 15 leaf traits of 49 Ethiopian mustard genotypes

\begin{tabular}{|c|c|c|c|c|c|}
\hline no & Character & Genotype(48) & Block(12) & Replication(1) & $\begin{array}{l}\text { Intra Block } \\
\text { error(36) }\end{array}$ \\
\hline 1 & Date of flowering & $141.98 * *$ & 6.39 & 0.91 & 9.96 \\
\hline 2 & Seed yield per plot & $503441^{*}$ & 15.9527 & 88.2551 & 9.6692 \\
\hline 3 & Plant height & $1004.12 * *$ & 1102.13 & 2812.50 & 1004.12 \\
\hline 4 & $\begin{array}{l}\text { Petiole length from bottom of } \\
\text { plant parts }\end{array}$ & $17.0989 * *$ & 3.3784 & 53.6352 & 3.3339 \\
\hline 5 & $\begin{array}{l}\text { Petiole length from middle of } \\
\text { plant parts }\end{array}$ & $12.1198 * *$ & 3.2264 & 32.5740 & 2.7985 \\
\hline 6 & $\begin{array}{l}\text { Petiole length from top of plant } \\
\text { parts }\end{array}$ & $9.0237 * *$ & 2.3248 & 15.4409 & 4.1043 \\
\hline 7 & $\begin{array}{l}\text { Leaf length from bottom of plant } \\
\text { parts }\end{array}$ & $10.0723 * *$ & 2.4737 & 20.2066 & 3.0007 \\
\hline 8 & $\begin{array}{l}\text { Leaf length from middle of plant } \\
\text { parts }\end{array}$ & $6.8706^{* *}$ & 1.8345 & 18.5180 & 2.5594 \\
\hline 9 & $\begin{array}{l}\text { Leaf length from top of plant } \\
\text { parts }\end{array}$ & $3.8865^{* *}$ & 2.6045 & 28.1250 & 3.1752 \\
\hline 10 & $\begin{array}{l}\text { leaf width from bottom of plant } \\
\text { parts }\end{array}$ & $8.2355 * *$ & 1.6135 & 22.6368 & 2.1843 \\
\hline 11 & $\begin{array}{l}\text { Leaf width from middle of plant } \\
\text { parts }\end{array}$ & $6.9200 * *$ & 2.7802 & 20.6633 & 1.9212 \\
\hline 12 & Leaf width from top plant parts & $4.6029 \mathrm{~ns}$ & 2.1734 & 23.0230 & 3.0136 \\
\hline 13 & $\begin{array}{l}\text { leaf area from Bottom of plant } \\
\text { parts }\end{array}$ & $5.1346^{* *}$ & 323.22 & 565.44 & 265.54 \\
\hline 14 & $\begin{array}{l}\text { leaf area from middle parts of } \\
\text { plants }\end{array}$ & $5.1571 * *$ & 494.55 & 229.59 & 265.95 \\
\hline 15 & $\begin{array}{l}\text { leaf area from top parts of } \\
\text { plants }\end{array}$ & $2.76692 \mathrm{~ns}$ & 293.30 & 729.07 & 204.20 \\
\hline
\end{tabular}

\subsection{Analysis of Variance}

The data collected for traits of leaf on different parts of plants were subjected to analysis of variance (ANOVA) for simple lattice design. Analysis of variance was done using Proc lattice and Proc GLM procedures of SAS version 9.2, (SAS Institute, 2008). Analysis of variance (Table 4) for the considered traits was done using the model for lattice design as follows: 


$$
\operatorname{Yil}(j)=u+t i+r j+(b / r) l(j)+e i l(j)
$$

Where, $\operatorname{Yil}(j)$ is the observation of the treatment $i\left(i=1, \ldots . V, k^{2}\right)$, in the block $l(l=1, \ldots k)$ of the replication $j(j=1, \ldots, m)$;

$\mu$ is a constant common to all observations;

$t_{i}$ is the effect of the treatment $i$;

$\mathrm{rj}$ is the effect of the replication $\mathrm{j}$;

$(\mathrm{b} \mid \mathrm{r})_{1(\mathrm{j})}$ is the effect of the block 1 of the replication $\mathrm{j}$;

$e_{i l(j)}$ is the error associated to the observation $\mathrm{Y}_{\mathrm{il}(\mathrm{j}) \text {, where }} \mathrm{e}_{\mathrm{il}(\mathrm{j})} \sim \mathrm{N}(0, \mathrm{~s})$ independent.

Table4. Simple Lattice analysis of variance and expected mean squares

\begin{tabular}{|c|c|c|c|c|}
\hline Source of variation & $\mathrm{Df}$ & SS & MS & F-value \\
\hline Replication(r) & $\mathrm{r}-1$ & $\mathrm{SSr}$ & $\mathrm{MSr}$ & $\mathrm{MSr} / \mathrm{MSe}$ \\
\hline Genotype(g) & g-1 & $\mathrm{SSg}$ & MSg & $\mathrm{MSg} / \mathrm{MSe}$ \\
\hline Block within replication & $\mathrm{r}(\mathrm{b}-1)$ & $\mathrm{SSb}$ & $\mathrm{MSb}$ & $\mathrm{MSb} / \mathrm{MSe}$ \\
\hline Intra-block error & $(b-1)(r b-b-1)$ & $\mathrm{SSe}$ & MSe & \\
\hline Total & $\mathrm{rb} 2-1$ & SST & & \\
\hline
\end{tabular}

Where, Df $=$ degree of freedom, SS sum of squares; MS: mean of squares, $\mathrm{SSr}$ and MSr sum of squares and mean of replication, respectively: SSg and MSg are sum of squares and mean of genotypes, respectively: SSb and MSb are sum of squares and mean of blocks within replication respectively. SSe and MSe are sum of squares and mean of intera-block error.

\subsection{Estimation of Phenotypic and Genotypic Variability}

The variability present in the population was estimated by simple measures, namely range, mean, standard error, and phenotypic and genotypic variances and coefficients of variations. The phenotypic and genotypic variance and coefficients of variation was also estimated as per the procedure suggested by Burton and De Vane (1953) as follows:

$$
\begin{aligned}
& \delta^{2} p=\delta^{2} g+\delta^{2} e \\
& \delta^{2} g=\frac{M S g-M S e}{r}
\end{aligned}
$$

Where, $\delta^{2} g=$ Genotypic variance

$\delta^{2} P=$ Phenotypic variance

$\delta^{2} \boldsymbol{e}=$ Environmental (error) variance or Error mean square

$M S g$ = mean sum square due to genotypes (accessions)

$M S e$ =mean sum square of error (environmental variance)

$\mathrm{r}=$ number of replications

Phenotypic Coefficient of Variation (PCV), $P C V=\frac{\sqrt{\delta^{2} p}}{\bar{x}} x 100$

Genotypic coefficient of Variation (GCV), $G C V=\frac{\sqrt{\delta^{2} g}}{--} \times 100$ 


$$
\bar{x}=\text { Population mean of the character being evaluated }
$$

\subsection{Heritability (in broad sense)}

Heritability in the broad sense for quantitative characters was computed using the formula suggested by Singh and Chaudhary (1985):

$$
H=\frac{\delta^{2} g}{\delta^{2} p} \times 100
$$

Where, $\mathrm{H}=$ heritability in the broad sense.

$$
\begin{aligned}
& \left(\delta^{2} g\right)=\text { Genotypic variance and } \\
& \left(\delta^{2} p\right)=\text { Phenotypic variance }
\end{aligned}
$$

\subsection{Expected genetic advance (GA)}

The genetic advance (GA) for selection intensity $(\mathrm{K})$ at $5 \%$ was calculated by the formula suggested by Allard (1999) as:

$$
G A=K * \delta_{P} * H
$$

Where, GA $=$ expected genetic advance, $\delta_{p}$ =phenotypic standard deviation on mean basis, $\mathrm{H}=$ Heritability in broad sense, $\mathrm{K}=$ selection differential ( $\mathrm{k}=2.06$ at $5 \%$ selection intensity)

Genetic advance (as percent of mean) (GA) was computed to compare the extent of predicted genetic advance of different traits under selection using the formula:

$$
G A M=\frac{G A}{\bar{X}} * 100
$$

Where, ${ }^{-\bar{x}}=$ population mean of the quantitative character, GAM =genetic advance as percent of mean.

\subsection{Analysis of Genetic Parameters}

\section{Genotypic and Phenotypic Coefficient of Variation}

Estimates of genotypic and phenotypic variances, genotypic coefficient of variation (GCV), phenotypic coefficients of variation (PCV), heritability in broad sense, expected genetic advances and genetic advances as percent mean are given in Table5. Estimated genetic variance ranged from $0.3557 \%$ for leaf length from top of plant parts to 135887 for seed yield per plot (Table5). Likewise phenotypic variance ranged from $7.0617 \%$ for leaf length from top of plant parts to $735108 \mathrm{~kg} / \mathrm{ha}$ for seed yield per plot. Phenotypic coefficients of variation ranged from $13.254 \%$ for date of $50 \%$ flowering to 68.995 $\%$ leaf width from top parts of plants. Genotypic coefficients of variation ranged from $8.169 \%$ for leaf length of top of plants to $30.90 \%$ for petiole length from top parts of plants. seed yield per plot(735108, 135887 ),Plant height $(1173.58,417.33)$, leaf area from bottom parts of plants $(1025.39,247.16)$,date of $50 \%$ flowering $(151.945,66.01)$ petiole length from bottom parts of plants $(20.43,6.883)$, petiole length from middle parts of plants $(14.92,4.661)$ and leaf length of from bottom of plant parts $(13.07,3.536)$ showed high phenotypic and genotypic variances, respectively indicating that the genotypes could be reflected by the phenotype and the effectiveness of selection based on the phenotypic performance for these traits.

Low genotypic variance as compared to environmental variance was recorded for traits such as leaf length from top parts of plants (0.356) and leaf area from top parts of plants (0.658), leaf length from middle parts of plants (2.1556) and petiole length from top parts of plants (2.6821). However, high genotypic coefficients of variation (GCV) and phenotypic coefficients of variation (PCV) were shown 
in traits such as petiole length of from top of plant parts(30.900, 67.195), petiole length of from middle of plant parts $(29.477,52.195)$, leaf width from middle of plant parts $(26.05,49.474)$, leaf width from top of plant parts $(22.286,68.995)$, leaf area from middle parts of plants $(24.908,45.257)$ and leaf area from bottom parts of plants $(22.192,42.022)$ respectively, which means selection of these traits based on phenotype characteristics may be useful for assessment of leaf variability in different parts of plant for improvement leaf traits.

Heritability in the broad sense

Breeders can make rapid progress where heritability is high by using selection methods that are dependant solely on phenotypic characteristics (e.g. mass selection). However, where heritability is low methods of selection based on families and progeny testing are more effective and efficient. Heritability estimated using the total genetic variance is called broad sense heritability .Heritability in the broad sense of the traits is presented in Table 5. In this study, heritability values were found to be sufficiently high for most important yield component characters. Dabholkar (1992) generally classified heritability estimates as low (5-10\%), medium (10-30\%) and high (30-60\%). Based on this classification, date of $50 \%$ flowering (43.442\%), plant height (35.560), petiole length from bottom of plant parts $(33.684 \%)$, petiole length from middle of plant parts $(31.241 \%)$, leaf width from bottom of plant parts $(30.134 \%)$ and leaf width from middle of plant parts $(30.291 \%)$ exhibited high heritability estimates. On the other hand leaf width from middle of plant parts(28.270\%), leaf area from bottom of plant parts $(27.889 \%)$, leaf length from bottom of plant parts(27.047\%), leaf length from middle of plant parts(22.859), seed yield per plot (18.485),leaf area from top of plants(15.59\%)and leaf width from top of plants(10.433\%) exhibited medium heritability estimates while only leaf length from top of plant parts $(5.036 \%)$ exhibited low heritability estimates. Date of $50 \%$ flowering was found to be the most heritable trait in the genotype, with heritability of $43.442 \%$, followed by plant height $(35.56 \%)$, petiole length from bottom of plant parts $33.684 \%$. Except only leaf length from top of plant parts all leaf traits showed high and medium heritability estimates. This indicates that selection for these traits in the genotype would be most effective for the expression of these traits in the succeeding generations.

Therefore, good improvement can be made if some of these traits are considered as selection criteria in future leaf traits improvement program. Similar findings had been reported by Jane Muthoni (2010) for leaf number/plant, leaf bloom and leaf blade blistering, flowering time, plant height, and seed yield per plant. High heritability value for thousand seed weight and seed yield per plot recorded in the current study was also recorded by Yared (2010) and Abebe (2006).According to Singh (1993), if the heritability of a character is high, selection for such character is fairly easy as selected character will be transmitted to its progeny. This is because there would be a close correspondence between the genotype and phenotype due to a relatively similar contribution of the environment to the genotype.

\section{Genetic advance}

Concerning the genetic advance at 5\% intensity the highest genetic gain was predicted for seed yield per plot (326.490\%), plant height $(25.095 \%)$ followed date of $50 \%$ flowering $(11.031 \%)$ and while the lowest genetic advance was predicted for leaf length from top plant parts $(0.276 \%)$. Genetic advance as a percent mean ranged from $3.77 \%$ for leaf length from top of plant parts to $35.242 \%$ for petiole length from bottom of plant parts (Table 5). Within this range, a relatively high genetic advance as a percent mean was observed for petiole length from bottom of plant parts $(35.242 \%)$ and petiole length from middle of plan parts $(33.591 \%)$ followed by petiole leaf length from top of plant parts(29.272). On the other side high genetic advance with medium heritability was shown for seed yield per plot $326.490 \%$ which may be because of the presence of both additive and non-additive gene action (Liang et al., 1972). On the other hand, the lowest genetic gain as percent of means was observed for leaf length from top of plant parts $3.777 \%$ and date of $50 \%$ date of flowering $11.861 \%$.. Low genetic advance as percent means observations in this study indicates that characters probably were under environmental influence than the genotypic expression and that selection based on these traits would be ineffective.

Table5. Components of variance, coefficients of variability, heritability and genetic advance and Genetic advance as percent of mean of studied traits

\begin{tabular}{|l|c|c|c|c|c|l|l|l|l|}
\hline Character & Mean & $\delta^{2} \mathrm{~g}$ & $\delta^{2} \mathrm{e}$ & $\delta^{2} \mathrm{ph}$ & $\mathrm{GCV}$ & $\mathrm{PCV}$ & $\mathrm{h}^{2} \mathrm{~b}$ & $\begin{array}{l}\text { GA } \\
\mathrm{k}=5 \%\end{array}$ & $\begin{array}{l}\text { GA/Grand } \\
\text { mean } \\
* 100 \mathrm{k} 5 \%\end{array}$ \\
\hline
\end{tabular}


Leaf Traits Variability of Ethiopian Mustard (Brasica Carinata A. Braun) Landraces in their Different Plant Parts

\begin{tabular}{|c|c|c|c|c|c|c|c|c|c|}
\hline $\begin{array}{ll}\text { Date } & \text { of } \\
\text { flowering }\end{array}$ & 93 & 66.01 & 85.93 & 151.94 & $8 . .736$ & 13.254 & 43.442 & 11.031 & 11.86 \\
\hline $\begin{array}{l}\text { Seed yield per } \\
\text { plot }\end{array}$ & 1999 & 135887 & 59921 & 735108 & 18.441 & 42.891 & 18.485 & 326.49 & 16.33 \\
\hline Plant height & 181 & 417.33 & 756.25 & 1173.58 & 11.28 & 18.927 & 35.56 & 25.09 & 13.86 \\
\hline $\begin{array}{l}\text { Petiole length } \\
\text { from bottom of } \\
\text { plant parts }\end{array}$ & 8.9 & 6.8825 & 13.55 & 20.4328 & 29.14 & 50.789 & 33.68 & 3.13 & 35.242 \\
\hline $\begin{array}{l}\text { Petiole length } \\
\text { from middle of } \\
\text { plant parts }\end{array}$ & 7.4 & 4.6607 & 10.26 & 14.9183 & 29.57 & 52.195 & 31.24 & 2.48 & 33.591 \\
\hline $\begin{array}{l}\text { Petiole length } \\
\text { from top of } \\
\text { plant parts }\end{array}$ & 5.3 & 2.6821 & 10.00 & 12.6832 & 50.15 & 67.195 & 21.15 & 1.55 & 29.272 \\
\hline $\begin{array}{l}\text { Leaf length } \\
\text { from bottom of } \\
\text { plant parts }\end{array}$ & 9.5 & 3.5358 & 9.54 & 13.073 & 18.8 & 38.06 & 27.05 & 2.01 & 21.205 \\
\hline $\begin{array}{l}\text { Leaf length } \\
\text { from middle of } \\
\text { plant parts }\end{array}$ & 8.7 & 2.1556 & 7.27 & 9.43 & 16.31 & 35.297 & 22.86 & 1.44 & 16.621 \\
\hline $\begin{array}{l}\text { Leaf length } \\
\text { from top of } \\
\text { plant parts }\end{array}$ & 7.3 & 0.3557 & 6.71 & 7.0617 & 8.52 & 36.403 & 5.036 & 0.56 & 3.777 \\
\hline $\begin{array}{l}\text { leaf width } \\
\text { from bottom of } \\
\text { plant parts }\end{array}$ & 7.1 & 3.097 & 7.18 & 10.2771 & 25.14 & 45.152 & 30.13 & 1.98 & 28.029 \\
\hline $\begin{array}{l}\text { Leaf width from } \\
\text { middle of plant } \\
\text { parts }\end{array}$ & 6.01 & 2.4994 & 6.34 & 8.8412 & 28.09 & 49.474 & 28.27 & 1.73 & 28.812 \\
\hline $\begin{array}{l}\text { Leaf width } \\
\text { from top plant } \\
\text { parts }\end{array}$ & 4 & 0.7947 & 6.82 & 7.61652 & 22.28 & 68.995 & 10.43 & 0.59 & 14.829 \\
\hline $\begin{array}{l}\text { leaf area from } \\
\text { Bottom of plant } \\
\text { parts }\end{array}$ & 6.1 & 1.839 & 4.751 & 6.59 & 22.192 & 42.022 & 27.889 & 1.4275 & 24.142 \\
\hline $\begin{array}{l}\text { leaf area from } \\
\text { middle parts of } \\
\text { plants }\end{array}$ & 5.6 & 1.946 & 4.474 & 6.42 & 24.908 & 45.257 & 30.291 & 1.581 & 28.240 \\
\hline $\begin{array}{l}\text { leaf area from } \\
\text { top parts of } \\
\text { plants }\end{array}$ & 4.2 & 0.658 & 3.562 & 4.22 & 19.495 & 49.372 & 15.591 & 0.660 & 15.857 \\
\hline
\end{tabular}

variance, $\delta^{2} e=$ Error variance, $\delta^{2} p h=$ Phenotypic variance, $G C V=$ Genotypic coefficient of variability, $P C V=$ Phenotypic coefficient of variability, $h 2 b=$ Broad sense heritability, $G A=$ Genetic advance and $K=$ Selection intensity

\section{CONClusion}

In this study, 49 Ethiopian mustard genotypes acquired from diverse zones/regions of Ethiopia were evaluated in simple lattice design with two replications at Holetta Agricultural Research Center, West Shewa zone, with the objective of assessing traits of leaf variability in their different plant parts of Ethiopian mustard land races in relation to high quantity and quality of leaf production traits. The analysis of variance showed the presence of significant differences among genotypes for all leaf parameter in their bottom, middle and top different plant parts of leaf traits except leaf width and leaf area from top plant parts compared. The significant difference indicates the existence of genetic variability among the accessions which is important for leaf traits improvement. Likewise phenotypic variance ranged from $4.22 \%$ for leaf area from top of plant parts to $735108 \%$ for seed yield per plot. Phenotypic coefficients of variation ranged from $13.254 \%$ for date of $50 \%$ flowering to $68.995 \%$ leaf 
width from top parts of plants. Genotypic coefficients of variation ranged from $8.169 \%$ for leaf length from top of plant parts to $30.900 \%$ for petiole leaf length from top parts of plants.

High phenotypic coefficient of variation (PCV) was recorded for leaf width from top parts of plant $68.995 \%$, petiole leaf length from top parts of plant $67.195 \%$ and leaf length from middle parts of plants 52.195\%.. But low PCV was detected for date of $50 \%$ flowering and plant height. Generally, the magnitudes of phenotypic coefficient of variation (PCV) and genotypic coefficient of variation (GCV) were high for leaf width from top of plants, petiole leaf length from top of plant parts and petiole length from middle of plant parts. Heritability in broad sense estimates was high for date of $50 \%$ flowering, plant height, petiole length from bottom of plant parts, petiole length from middle of plant parts, leaf width from middle of plant parts. On the other hand leaf width from middle of plant parts, leaf area from bottom of plant parts, leaf length from bottom of plant parts, leaf length from middle of plant parts, seed yield per plot, leaf area from top of plants and leaf width from top of plants exhibited medium heritability estimates while only leaf length from top of plant parts exhibited low heritability estimates .Genetic advance as percent of the mean (GAM) was high for petiole length from bottom of plant parts petiole length from middle of plant parts, petiole length from top of plant parts and while the lowest genetic advance was predicted for leaf length from top plant parts $(0.276 \%)$. Genetic advance as a percent mean ranged from $3.777 \%$ for leaf length from top of plant parts to $35.242 \%$ for petiole length from bottom of plant parts (Table 5). Within this range, a relatively high genetic advance as a percent mean was observed for petiole length from bottom of plant parts(35.242\%) and petiole leaf length from middle of plan parts $(33.591 \%)$ followed by leaf width from middle of plant parts(28.812). On the other side high genetic advance with medium heritability was shown for seed yield per plot and plant height which may be because of the presence of both additive and non-additive gene action. These results indicate that there is good opportunity to improve leaf variability traits using the tested genotypes.

\section{REFERENCES}

[1] Abebe Delesa. 2006. Genetic Variability and Association Among Seed Yield and Yield Related Traits in Ethiopian mustard (Brassica carinata A. Braun) at Kulumsa, Arsi. An M.Sc. Thesis Presented to the School of Graduate Studies of Alemaya University. 75p.

[2] Adefris Teklewold. 2005. Diversity Study Based on Quality Traits and RAPD Markers and Investigation of Heterosis in Ethiopian Mustard. Ph.D. diss. Georg-August Univ. of Göttingen, Germany. 161p.

[3] Allard, R.W. 1999. Principles of plant breeding. 2thed. New York, John Wiley \& Sons, 254 p. ISBN 978-0471-02309-8.

[4] Burton, G.W. and E.H. de Vane. 1953. Estimating heritability in tall fescue(Festuca

[5] arundinacea) from replicated clonal material. Agron. J. 45: 478-481

[6] Doweny R.K. and G. RÖbbelen. 1989. Brassica Species. In RÖbbelen G, Doweny RK and Ahri A (eds) Oil crops of the world. McGraw-Hill New York. pp. 339-359

[7] Gomez-Campo, C. and S. Prakash. 1999. Origin and domestication of the Brassica. pp. 33-58. In: GomezCampo C (ed.). Biology of Brassica Coenospecies. Elsevier, Amsterdam.

[8] Hemingway, J.S. 1976. Mustards Brassica species and Sinapsis alba (Cruciferae). In: Evolution of Crop Plants. N.W. Simmounds (ed.) Longan. London. 339p.

[9] Jane Muthoni.2010.Characterization of Ethiopian Mustard(Brassica carinata A. Braun)lines for vegetative agromorphological traits at Arusha,Tanzania.Jornal of Horti and forestry Vol.2(1)pp.002006

[10] Liang, G.H., C.R. Reddy and A.D. Dayton.1972. Heterosis, inbreedign depression and heritability estimates in a systematic series of grain sorghum genotypes. Crop Sci. 12(4):409-411

[11] Neeru, N. K. Thakral, Ram Avtar and Hari Kesh 2017.Journal of Applied and Natural Science 9 (2): 805 811 (2017)

[12] Nigussie Alemayehu. 2001. Germplasm diversity and Genetics of Quality and Agronomic Traits in Ethiopian Mustard (Brassica carinata A. Braun). Ph.D. Thesis, George-August University of Gottingen, Germany

[13] Nigussie Alemayehu and Mesfin Abebe. 1994. Relative importance of some managmnet factors in seed and oil yields of Ethiopian mustasrd (Brasica carinata Braun.) and Rapeseed (Brasica napus L.). Ethiopia. J. Agric. Sci. 14: 27-36

[14] SAS Institute INC., 2002- 2008. SAS*STAT, users guide, version 9.2, Cary N.C., SAS INC

[15] Singh, B. D. 1993. Plant breeding principles and methods. Kalyani Publishers, Ludhiana, New Delhi. 
[16] Singh, R.K. and B.D. Chaydhary. 1985. Biometrical methods in quantitative genetic analysis

[17] U.N. 1935. Genome analysis in Brassica with special reference to the experimental formation of B. napus and peculiar mode of fertilization. Jpn. J. Bot. 9: 389-452

[18] www. Seed laboratory Oregon state university. Oil, Protein and moisture Determination using NMR

[19] Yared Semahegn. 2010. Genetic diversity and Relationship among Association among Ethiopian mustard (Brassica carinata A. Braun) genotypes based on their agronomic and quality Traits. M.Sc. Thesis Presented to the School of Graduate Studies of Jima University. 75p.

[20] Rabbani, M. A., A. Iwabuchi, Y. Murakami, T. Suzuki and K. Takayanagi.1999. Collection, Evaluation and Utilization of Oilseed Mustard (Brassica juncea L.) in Pakistan.Pakistan Journal of Biological Sciences, 2 (1): 88-94, 1999

[21] Revilla, P. and W.F. Tracy, 1995.Morphological characterization and classification of open-pollinated sweet corn cultivars. J. Am. Soc. Hortic. Sci., 120: 112-118.

Citation: Fekadu Amsalu., "Leaf Traits Variability of Ethiopian Mustard (Brasica Carinata A. Braun) Landraces in their Different Plant Parts" International Journal of Research Studies in Agricultural Sciences (IJRSAS), 2020; 6(12), pp. 12-20, 28-39 https://doi.org/10.20431/2454-6224.0612004

Copyright: (๑) 2020 Authors. This is an open-access article distributed under the terms of the Creative Commons Attribution License, which permits unrestricted use, distribution, and reproduction in any medium, provided the original author and source are credited. 